\title{
Editorial
}

\section{Orthotics in Neurorehabilitation}

Brain damage may lead to a range of disabilities, one of the most common being loss of movement. Some recovery of movement typically occurs, however often the complications of muscle contracture, spasticity, and/or sensory loss limit rehabilitation and reduce functional outcomes. Neurorehabilitation aims to enhance neural repair or development or compensate for limitations so that everyday activities can be performed as efficiently and effectively as possible.

The focus on function in neurorehabilitation necessarily means complex interventions are used to address body structure, activity, participation and environmental issues that may arise. Orthotics, like any tool used in the treatment of a complex and chronic condition, can target all levels of health at once. It may be an intervention designed to change body structures, or an intervention to support and stabilize unresponsive muscles so an activity can be performed, or an adjunct to enable participation in a life role such as work. To find out whether or not an orthosis is 'effective' its performance needs to be measured against indicators relevant to the level of health being targeted. Traditionally, orthotic effectiveness has been considered from the point of view of amelioration of impairment - in other words the ability of the orthosis to achieve change in body structures or functions such as pain, range of movement or spasticity. In neurorehabilitation, it is becoming increasingly clear that prescription and evaluation of orthoses must go beyond consideration of impairment indicators. It is, after all, activity and participation that matters most to people who live with a neurological condition and/or their families.

This special issue of NeuroRehabilitation on orthotics brings together leading researchers who have grappled with this challenge from different clinical and technical perspectives. With my colleague Professor Ada we describe the clinical reasoning processes used when providing orthotics in neurorehabilitation, and we make practical recommendations for clinical and research decision-making. The impact of orthoses on functional outcomes is further explored by Tyson and Kent, who examine whether or not wrist/fingers and/or thumb orthoses were effective in changing impairment indicators (pain, range of motion, spasticity) and upper limb function for stroke survivors. They used the rigorous Cochrane systematic review methodology to synthesize best available evidence. They found that not only did use thermoplastic orthoses post-stroke make no difference in impairment or function to those receiving usual treatment, but it made no difference when compared to placebo or no treatment conditions. Since splints are routinely prescribed in many stroke rehabilitation centres for these purposes, this review finding will be startling to many practitioners.

In another diagnostic group, children with cerebral palsy, Elliot and colleagues show that a dynamic lycra upper limb splint had a significant effect on both function and kinemetric indicators when used in a goaldirected training program. Elliot's strong finding is important when seen in the context of currently available research on orthotic use in cerebral palsy. Morris et al. present their appraisal of research evidence for orthotic practice in cerebral palsy using a consensus conference approach. This technique is commonly used in the development of clinical guidelines when available research is scant, patchy or as they discovered, fraught with methodological or reporting limitations. Their careful appraisal provides practitioners with research based management recommendations for ankle-foot orthoses to enhance gait efficiency, at the same time as filling the evidence gaps they found with expert panel recommendations on orthoses for hip, spine or upper limbs in cerebral palsy.

Given the patchy and contradictory nature of research evidence relating to orthotic use in neurorehabiliation it is worth considering how and why the practice of splinting was adopted? Harvey and colleagues overview the history of beliefs and practices relating to orthotic prescription for people with spinal cord injury. In a nutshell, orthoses were assumed to 
help prevent contractures - but Harvey et al. point out in their review that the research to date does not indicare that orthoses prevent this complication. The contrast of beliefs, practice and research evidence in spinal cord injury is an excellent case study for the type of orthotic practice issues now arising in a range of neurorehabilitation settings.

Finally, Blakey and Hoffman remind us that possibilities for prescription and manufacture open up as orthotic technology changes. Orthoses only imagined years ago are now available for routine prescription and use by people with significant neurodisability. Orthosis manufacture must be informed by, not only an understanding of impairments and activity limitations, but also by an understanding of individual functional goals so that newer technologies can be used and effectiveness in goal achievement measured.

Where to from here? The use of orthotics in neurorehabilitation is in a state of flux. Enormous oppor- tunities are opening with technological advances and the shift in focus to considering function and activities in the design, prescription and evaluation of orthoses in the context of goal directed programs. On the other hand, there is a growing body of evidence in some clinical areas such as stroke, that orthoses have no effect on target impairments such as spasticity, pain or range of motion or on function. As a close to this special edition, the focus is thus on the practitioner who must make decisions regarding orthotic use in neurorehabilitation.

Guest Editor

Natasha Lannin, PhD, BSc(OT), GradDip

Rehabilitation Studies Unit Sydney Medical School The University of Sydney Australia E-mail: Natasha.Lannin@sydney.edu.au 